\title{
Distributed Power Control in Ad-hoc Wireless Networks
}

\author{
Sharad Agarwal \\ CS Division, University of California, Berkeley \\ Randy H. Katz \\ CS Division, University of California, Berkeley
}

\author{
Srikanth V. Krishnamurthy \\ CSE Department, University of California, Riverside \\ Son K. Dao \\ ISL, HRL Laboratories, LLC
}

\begin{abstract}
Mobile ad-hoc networking involves peer-to-peer communication in a network with a dynamically changing topology. Achieving energy efficient communication in such a network is more challenging than in cellular networks since there is no centralized arbiter such as a base station that can administer power management. In this paper, we propose and evaluate a power control loop, similar to those commonly found in cellular CDMA networks, for ad-hoc wireless networks. We use a comprehensive simulation infrastructure consisting of group mobility, group communication and terrain blockage models. A major focus of research in ad-hoc wireless networking is to reduce energy consumption because the wireless devices are envisioned to have small batteries and be incapable of energy scavenging. We show that this power control loop reduces energy consumption per transmitted byte by $10-20 \%$. Furthermore, we show that it increases overall throughput by $15 \%$.
\end{abstract}

\section{INTRODUCTION}

Ad-hoc wireless networking is receiving renewed attention. It enables many interesting usage scenarios but poses several challenges. Traditionally, wireless networking has been applied to cellular telephony and Internet connectivity via radio modems. These systems provide single hop connectivity to a fixed, wired base station. Ad-hoc wireless network systems attempt to form multi-hop networks without pre-configured network topologies. There is peer-to-peer interaction among nodes, unlike in cellular networks where nodes communicate with a centralized base station. Ad-hoc networks are characterized by dynamically changing topologies, a direct result of the mobility of the nodes. Such systems can offer many advantages. They do not rely on extensive and expensive installations of fixed base stations throughout the usage area. With the availability of multiple routes to the same node or base station, they can perform route selection, based on various metrics such as robustness and energy cost. Nodes can communicate directly with each other when possible, rather than using a distant, intermediate base station. This can help conserve energy and improve throughput. These systems enable various applications, ranging from the monitoring of herds of animals to supporting communication in military battlefields [1] and civilian disaster recovery scenarios.

Many of these applications require that nodes be mobile and be deployed with little network planning. The mobility of nodes limits their size, which in turn limits the energy reserves available to them. Thus energy conservation is a key requirement in the design of ad-hoc networks. In wireless networks, bandwidth is precious and scarce. Simultaneous transmissions in domains which use the same bandwidth interfere with each other. Thus bandwidth re-use is also important.

This work was supported by DARPA (Defense Advanced Research Projects Agency) Contract No. N00014-99-C-0322.
Power control helps combat long term fading effects and interference. When power control is administered, a transmitter will use the minimum transmit power level that is required to communicate with the desired receiver. This ensures that the necessary and sufficient transmit power is used to establish link closure. This minimizes interference caused by this transmission to others in the vicinity. This improves both bandwidth and energy consumption. However, unlike in cellular networks where base stations make centralized decisions about power control settings, in ad-hoc networks power control needs to be managed in a distributed fashion.

In this paper, we present a power control loop for ad-hoc wireless networks. We describe the details of this algorithm in Section II. In Section III we describe the simulation infrastructure that we have built to simulate realistic ad-hoc networks. We have made an effort to model the node mobility, communication traffic and environment likely to be experienced in typical scenarios. We evaluate our power control loop in Section IV. Our power control loop improves energy consumption and throughput by $10-20 \%$ and $15 \%$ respectively in our simulation models.

\section{Distributed Power Control For AD-HoC COMMUNICATION}

In this section, we describe an energy conservation technique at the MAC layer. The goal here is to minimize the energy cost of communication between any given pair of neighboring nodes if such communication is possible. Ad-hoc networks can contain nodes of various types, of which many can have limited power capabilities and may not be able to scavenge energy from sources such as solar energy. Furthermore, many of the data gathering applications for which these networks are deployed are latency tolerant. Thus, energy efficiency rather than latency should be the principle design goal in MAC communication.

One main mechanism for energy conservation at the MAC layer is power control. Power control loops for various cellular telephony systems have been studied extensively in the past and are used in commercially deployed systems [2], [3]. They are especially important in ad-hoc networks due to the higher levels of interference. We have applied power control extensions to the IEEE 802.11 $\mathrm{MAC}^{1}$ specification [5], thereby achieving lower energy consumption and higher throughput.

In this section, we begin by describing the general concept behind power control and refer to related work. In a following subsection, we describe the IEEE 802.11 MAC protocol, which

\footnotetext{
${ }^{1}$ Neither the IEEE 802.11 specification nor its commercial implementations such as WaveLAN [4] use power control.
} 
is the MAC protocol we use for implementing power control. We then describe our distributed power control loop.

\section{A. Power Control}

In cellular systems, a base station tells mobile units to adjust their transmit powers by measuring the power received from them. Cellular systems are used for applications such as telephony where the pre-installation of a fixed base station infrastructure is feasible. Cellular systems have star topologies and every mobile unit communicates exclusively with an associated base station.

An ad-hoc network on the other hand does not have a centralized arbiter which can tell each node the transmit power to use to communicate with a particular receiver. Furthermore, well defined cells or domains do not exist. Thus power control in an ad-hoc network is not trivial and needs to be administered in a distributed manner. However, the benefits of power control remain. Instead of every node using the same transmit power, if a node uses only the power level that is required to communicate with a desired receiver, it might extend it's battery life. Furthermore, it will reduce interference seen by other simultaneous transmissions in the network.

\section{B. Related Work: Power Control Loops in Cellular Networks}

Power control loops for various cellular telephony systems have been studied extensively in the past and are used in commercially deployed systems [2], [3]. The related literature is vast, and we will not attempt a complete survey. Instead, we describe the basic concept behind power control loops in CDMA systems.

One of the main goals of power control is to avoid the nearfar effect. Since transmitted signals experience propagation loss, signals received by a base station from a closer mobile station will be stronger than those received from one that is further away. Thus distant mobile stations will not experience a fair share of the available throughput to the base station. Similarly, another goal of power control is to reduce the interference that a mobile station experiences from different base stations near the edge of a cell. In spread spectrum networks, especially in CDMA networks, power control is necessary to reduce the average noise level so that it is possible to recover the spread signal.

Both open loop and closed loop power control mechanisms have been explored in CDMA systems. Open loop control attempts to measure, at the mobile station, the path loss between itself and the base station. Using the received signal strength of messages and various control parameters transmitted by the base station, the mobile station can set its transmit power level. This mechanism does not always achieve the best transmit power level because the path loss experienced on the uplink and downlink may differ (especially if different frequencies are used for the uplink and downlink).

Closed loop power control treats uplink and downlink power control separately. The base station measures the received signalto-interference ratio (SIR) over a short time period and decides whether the mobile station should raise or drop its transmission power level by comparing the received SIR to the appropriate SIR value. This decision is transmitted to the mobile station on the downlink. The mobile station then adjusts it's transmit power levels accordingly. The base station determines the optimal SIR value by an outer control loop that considers the error rate experienced on the uplink. CDMA systems use a similar closed loop power control to adjust the downlink transmit power levels. The base station periodically reduces it's transmit power levels. The mobile station measures the error rate experienced on the downlink and requests additional power from the base station if the experienced error rate is unacceptable. The downlink control loop iterates at a frequency that is at least an order of magnitude lower than the uplink control loop.

Reference [6] in particular describes an adaptive closed loop power control algorithm for cellular CDMA networks that is similar to the one we propose in this paper for ad-hoc networks. Their simulations of cellular CDMA networks consist of hexagonal cell layouts with each cell consisting of randomly moving nodes that communicate only with base stations.

\section{Related Work: Power Control Loops in Ad-Hoc Networks}

Ad-hoc wireless networks provide a different set of challenges than standard cellular telephony and packet radio networks. We cannot placed the network in a pre-surveyed cellular fashion. Each node communicates directly with many nodes rather than just one base station. Reference [7] attempts to impose a cellular structure to an ad-hoc network topology. Each cluster head acts like a cellular base station. The authors propose to use open loop and closed loop power control in a similar fashion as described above in cellular networks, but specifically to control the size of a cluster. Their main goal is to reduce the number of network topology changes that occur as a function of node velocity.

Reference [8] is similar to [7] but formulates the problem differently, as an optimization problem. The authors determine the "optimal" number of neighbors that each node should have to minimize the maximum transmit energy while maintaining connectivity constraints. In the algorithms that they propose, they restrict the number of neighbors of a node by reducing its transmit power level by a constant value.

The power control loop mechanism that we propose is different from prior work in several ways. Our focus is on ad-hoc networks and not cellular systems as in references [2], [3], [6], [7]. We allow each node to choose different transmit power levels for different neighboring nodes. It is not a goal of the work we present here to reduce the connectivity of the nodes (as in references [7], [8]). We allow all nodes to communicate with all of their neighbors, but by having each node choose different transmit power levels for each of its neighbors, interference will be reduced. A system that ties each node to a single transmit level for all communication (as in reference [8]) will experience higher interference because excessive transmit power will be spent communicating with nearby nodes. We investigate whether a power control loop in an ad-hoc wireless MAC can reduce energy consumption and increase overall throughput. We evaluate the performance of our scheme by means of constructing realistic simulation models that can depict various scenarios.

We have applied our power control loop to the IEEE 802.11 MAC [5]. In the following subsection, the relevant part of the IEEE 802.11 MAC specification is briefly described. We follow it by a description of the modifications we propose to support 


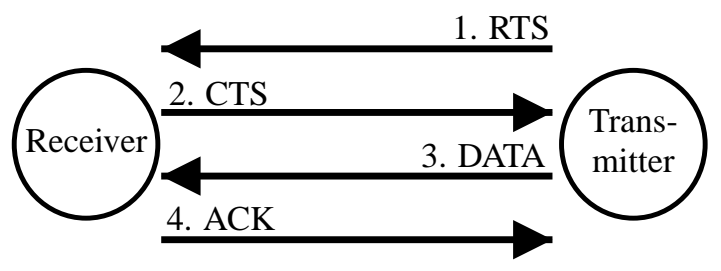

Fig. 1. IEEE 802.11 Signaling for Addressed Messages

power control.

\section{IEEE 802.11 MAC Signaling System}

We consider adding power control to ad-hoc wireless medium access to reduce energy consumption by reducing both the transmission energy and average RF (radio frequency) interference. We incorporate a power control algorithm into the IEEE 802.11 MAC protocol [5], which has been popular for ad-hoc networks [9]. The power control modifications that we propose in the next subsection involve piggy-backing additional control information in the IEEE 802.11 MAC's signaling. These modifications are applicable to any ad-hoc MAC protocol that employs a signaling scheme similar to the one specified in the IEEE 802.11 standard. In the remainder of this subsection, we provide an overview of the relevant parts of this standard.

There are two basic message types that the IEEE 802.11 MAC layer generates - (a) broadcast messages and (b) messages destined for a specific host within the node's radio range (hereby referred to as addressed messages). When the MAC layer of a node generates a broadcast message, which is destined for all hosts within the node's range, the node simply transmits the message without any additional signaling.

When a node has to transmit an addressed message, it uses a signaling protocol (RTS-CTS-DATA-ACK) (see Figure 1) that is more complicated than the one used for broadcasting. This protocol includes the generation of messages that inform the destination and other neighboring nodes about a forthcoming data transmission, and thus reduces the effects of hidden terminals [10]. These nodes will defer other transmissions during this period.

\section{E. Modifications to Incorporate Power Control}

We now describe our modifications to the IEEE 802.11 MAC specification for addressed messages to support power control. In the original IEEE 802.11 MAC, all transmissions occur at the same transmit power level. For our power control loop, we allow this transmit level to be any one of ten levels. These levels vary linearly between the default transmit power level (the maximum) and one-tenth of this value ${ }^{2}$. We scale down the energy to transmit a message by the transmit power level chosen by our power control loop. It is possible in an implementation that the reduction in energy consumption levels will not match the reduction in

\footnotetext{
${ }^{2}$ In comparison, AMPS (Advanced Mobile Phone System) uses eight power levels [11]. GSM (Global System for Mobile communications) uses eight to fifteen levels, depending on the unit's maximum transmitter power.
}

transmit power levels due to inefficiencies in it's design. We will explore the result of such issues in Section IV.

We also alter the message header formats for CTS and DATA messages to include a value which is the ratio of the received signal strength of the last received message to the minimum acceptable signal strength at the node currently transmitting the message. When a receiver receives an RTS message, it will encode the ratio of the received signal strength of the RTS message to the minimum signal strength that is acceptable by this receiver in the header of the CTS reply message. Similarly, when transmitting the DATA message, the transmitter will encode into it the ratio with respect to the received CTS. Thus, during one RTS-CTS-DATA-ACK exchange, both the transmitter and the receiver inform each other about the quality of their transmitted signals. Both nodes now have the opportunity to alter their transmit power levels for further communication between each other.

The MAC layer for each node maintains a small table that stores power control settings for other nodes with which this node has recently communicated. The table will be small since it is unlikely that a node will communicate directly with more than a few neighbors at any point in time. The table stores the current transmit power level setting used for each neighbor. The $\mathrm{cf}$ _pwr field of the table maintains an EWA (exponential weighted average) history of the received signal strength ratio received from each neighbor. The dr_pwr field maintains an EWA history of the cf_pwr field at instances when packet losses occurred. A count-down timer field is also maintained for each neighbor to dampen rapid fluctuations in transmit power levels.

When a message is sent to a node, we look up the node ID in the table. If it is not there, we allocate an entry with the initial power level set to maximum (see Section IV for simulation results with lower initial settings). When a CTS or DATA message is received from a node, we update it's cf_pwr field in the table. If the cf_pwr field is higher than the dr_pwr field, we decrement the transmit power level field by one, unless the count-down timer field is not null. When the MAC times out while waiting for a CTS or DATA or ACK message from a node, we increment the transmit power level field by one and update the dr_pwr field. We set the count-down field to ten. This ensures that for the next ten message transmissions to this node, the transmit power level field will not be decremented. We chose this value of ten to dampen rapid fluctuations while ensuring the overall effectiveness of the power control loop.

Our modifications apply to addressed messages between any pair of nodes. We have not extended the power control algorithm to broadcast messages where only a DATA message is transmitted. These messages are typically used for routing purposes and do not involve a sequence of message exchanges. It is not possible for the MAC layer to decide what transmit power level they should use since the destination is no longer a single node.

The essential goal of the above algorithm is to learn the minimum transmit power level required for a node to successfully transmit to a neighboring node. Starting with an initial value for the transmit power level, the exchange and loss of messages causes the MAC layer to ratchet up (or down) the transmit power level. The MAC layer of a node thus learns the unique minimum transmit power level required for that node to successfully trans- 
mit to any other nearby node. A lower level will result in lost packets. This level is unique for every node with this node communicates. This level can change, and so the algorithm continuously tracks the returned signal strength ratio and determines if the transmit power level should be changed. We use the simulation infrastructure that we describe in the next section to evaluate our power control algorithm in Section IV.

\section{Group Mobility Simulation Models}

To evaluate new algorithms for use in ad-hoc wireless networks, realistic usage patterns need to be employed. It is a common practice in published works to use random node placement, mobility and traffic patterns. These patterns do not accurately model real deployments of ad-hoc wireless networks. In deployed ad-hoc networks, there are many physical obstacles to radio waves. In the scenarios we consider, nodes do not move randomly, but move in accordance with coordinated groups. Traffic is not random, but represents a well defined flow of data and control between the nodes.

The mobility, traffic and blockage models that we present here can be used to model many real usage scenarios. Various animals (such as wolves, birds and fish) and wilderness explorers (such as hikers and skiers) tend to travel in groups. Environmentalists wishing to track the movements of these animals may attach radio transceivers to them. They can form an ad-hoc network, allowing various location and sensor readings to propagate to distant base stations. Law enforcement officers, military troops, fire fighters and medical personnel also move and work in groups. Modeling such environments using random patterns is inadequate for the evaluation of new networking algorithms. We must apply group mobility and traffic patterns and we must model the blockages that would be experienced in real usage.

\section{A. Related Work: Realistic Scenario Modeling}

Much of the research literature in ad-hoc wireless networking resorts to inaccurate and unrealistic random models. We summarize in this subsection various other models that have been proposed in the past.

One main usage scenario is the military battlefield of the future. Scattered troops and vehicles will need to communicate via a network formed in an ad-hoc fashion. Reference [12] provides an example of a hierarchical tactical military network control structure to motivate their work on the application of mobile IP and CIDR (Classless Inter-Domain Routing) to such networks. However, they do not present simulations of such node placement and communication patterns. Reference [13] uses a static arrangement of nodes in a tactical network, where nodes are part of different network groupings (cohorts). One member of each of the groupings is part of a larger grouping. They use this model in simulations, but without any mobility patterns or terrain models. Reference [14] presents a very detailed simulation of a tactical network. They support node mobility and the loss of nodes due to enemy fire and jamming attacks. However, the user has to specify mobility patterns and traffic probability distributions.

Reference [15] describes three different scenarios - users at a conference, users at a public event and monitoring a disaster area. They place nodes in clusters. The nodes move randomly

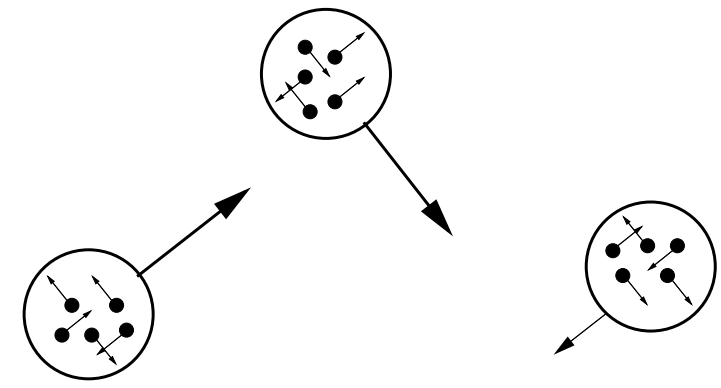

Fig. 2. Group Mobility

within a cluster but the clusters do not move. They place obstacles that do not move and completely block all transmissions through them. We present more sophisticated simulation models targeted at more general scenarios involving group mobility. We move nodes in their groups as a whole, while giving each node the same trajectory as their group but with a slight variance. Furthermore, reference [15] does not explain how traffic sources and destinations are assigned. We set up traffic flows in a hierarchical manner: flows among nodes within the same group and flows between groups. Our blockage models are more sophisticated in that they move and transmission loss is not absolute. It varies based on the loss characteristics of the obstacles and the nature of how the obstacle blocks the transmission.

Reference [7] presents a survey of various mobility models and investigates the impact of group mobility on the performance of various routing protocols. They conclude that random mobility models do not accurately predict the performance of routing protocols in real usage scenarios. The group mobility model that we present is similar to theirs. However, we additionally incorporate group communication patterns and blockage models to further improve the fidelity of our simulation results.

\section{B. Mobility Models}

To model group movement, we pre-generate motion vectors for each node and feed them into our simulations. The users of this model need to specify various high level parameters : the number of nodes to be simulated, the size of a group, the maximum speed of a node and a random number generator seed. Using this list of parameters, our simulation infrastructure generates motion vectors for individual nodes. We initially place the nodes with their groups, and place the groups at random within the simulated field of variable size. We give each group a randomly chosen trajectory and speed. This is random because more accurate models of group movements require profiles of a specific application, which are not currently available. All the nodes within that group follow this chosen trajectory and speed but with a small random variance. This small variance is meant to model real life effects, such as animals or people moving with varying speeds and application specific responsibilities. Once this motion vector has been followed for a certain random period of time, the group will pause for a short period and will choose another vector. The group pauses at each destination to simulate the fulfillment of an application specific goal such as the investigation of an area or the collection of sensor data. This model is shown in Figure 2. 


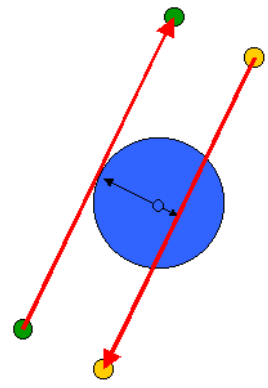

Fig. 3. Blockage Model

\section{Traffic Models}

The nodes (animals, humans or machines) within a group want to share sensor and location information so that local inferences about sensor readings can be made. Thus the traffic pattern will consist of intra- and inter- group communications of data and control. To model such traffic, we pre-generate intra-group and inter-group traffic patterns that we feed into our simulations. The resulting communication pattern will have roughly $0.75 * N$ connections distributed among the $N$ nodes in each group, with each connection starting at a random time. There will be roughly $0.75 * G$ connections between $G$ groups. The goal of this model is to mimic the connection patterns that are likely to be used in actual ad-hoc network deployments. We do not attempt to model the amount or rate of the actual data flow because we do not have traces of real usage.

\section{Blockage Models}

The areas where ad-hoc networks will be deployed will rarely consist of just open, flat terrain. It is probable that various forms of foliage, mountainous terrain, buildings, enemy RF jammers and inclement weather (rain, snow, hail) will be experienced. This harsh environment will manifest itself by impairing RF transmissions. We make an effort to model blockages so that our simulations will more accurately reflect realistic transmission effects.

We can accurately model every feature of every kind of blockage to infinite detail. However, it is important for us to maintain a balance between modeling accuracy and simulation run time. Thus we make one important simplification - we model all blockages as spheres of varying radii. This makes it easier to quickly determine whether the line of sight between two nodes intersects a blockage. Also, we need to avoid this calculation for every transmission for every receiver for every blockage. Instead, for every sender and receiver pair, we consider blockages near them. We internally store blockages in a multi-level quad cif tree representation of the simulation field. We pick those blockages in the smallest quadrant encompassing both the sender and the receiver. For mobile blockages, we re-arrange them at the appropriate times in the quad-cif tree. At present, we allow nodes to pass through blockages. This is accurate for heavy foliage or weather, but not for buildings or hills.

When we consider the effect of a blockage on a transmission, we first determine whether it is blocking the transmission. If it is, we drop the energy of the transmission by the equation below.
Each blockage has a certain loss factor (blockage density), $L$. The wavelength of the transmission is $\lambda$, the radius of the blockage sphere is $R$, the shortest distance between the center of the sphere and the point of intersection is $d$ and the energy of the transmission is $E$.

$$
E *\left(1-\left(\frac{\lambda * d}{4 * \pi * R}\right)^{2} * \frac{1}{L}\right)
$$

The above equation is an approximation of the true blockage loss that would occur to a transmission. The main features of the above equation are that the loss is greater the closer the intersection is to the center and the loss is greater the smaller the value of $L$. Thus, in Figure 3, the communication between the lighter colored nodes will incur a higher energy loss (interference) than that between the darker colored nodes. For each transmission, we consider blockages in no particular order. Furthermore, we apply the blockage losses after we account for the free space propagation loss in the energy of the transmission. Both of these approximations should have a minor impact on simulation accuracy.

\section{Simulation EnVironment AND RESUlts}

We have incorporated these group mobility, traffic and blockage models into our simulation environment. We use this environment to evaluate our distributed power control algorithm. While the emphasis of this paper is on realistic usage models, we also present results from completely random models to justify the need for more real usage models. In this section, we describe the simulation environment and the experimental setup using (a) the random models and (b) realistic models using the group mobility, traffic and blockage patterns. We then present our simulation results.

\section{A. Network Simulator}

We use the UCB/LBNL discrete event network simulator, NS (version 2.1b6) [16], which is now under development as part of the VINT project. We chose NS because of its CMU Monarch project extensions that support various ad-hoc routing protocols and its extensibility. The NS simulator contains an implementation of the IEEE 802.11 MAC standard [5] which executes above a wireless RF (radio frequency) physical layer. The physical layer is a model of a DSSS radio interface (Lucent WaveLan [4] Direct-Sequence Spread-Spectrum) operating at $914 \mathrm{Mhz}$ with a throughput of $244 \mathrm{KBps}$. We have modified the physical and MAC layers to support our power control loop algorithm.

\section{B. Simulation Setup of Random Models}

Our goal in presenting results of simulations using random models is to contrast them with those from real usage models. Here we list the simulation setup parameters that we use. In the random simulation models, we do not use any blockage models. We run each of the simulations with pre-generated node placement, movement pattern and traffic patterns. Initial node placement within the simulated field is random. We select random speeds and directions for each node at random times to generate movement patterns. For traffic patterns, we pick two nodes 
TABLE I

RANDOM Simulation MOdel SETUP PARAMETERS

\begin{tabular}{lr}
\hline Field Length & 500 meters \\
\hline Field Width & 500 meters \\
\hline Simulation Time & 10 seconds \\
\hline Traffic Type & TCP sessions \\
\hline \# of TCP Sessions & $2-28$ \\
\hline \# of Nodes & 30 \\
\hline Energy Budget / Node & 1 Joule \\
\hline Routing Protocol & DSR[17] \\
\hline Max Node Speeds & $1,10 \& 20 \mathrm{~m} / \mathrm{s}$ \\
\hline
\end{tabular}

TABLE II

Additional Group Mobility Simulation SETUP PARAMETERS

\begin{tabular}{lr}
\hline Nodes / Group & 5 \\
\hline \# of Blockages & 4 \\
\hline Max Blockage Radius & 10 meters \\
\hline Max Blockage Speed & $2 \mathrm{~m} / \mathrm{s}$ \\
\hline
\end{tabular}

at random, and at a random time, we initiate a TCP session. We vary the seeds used for the random number generators to produce a set of results that we average and show the highest and lowest values in error bars. ${ }^{3}$

Table I lists various simulation setup parameters. The energy budget of 1 Joule allows each node to transmit and/or receive about $800 \mathrm{~KB}$ at full transmit power. Most nodes die of energy starvation by the end of each simulation which lasts 10 seconds. When nodes run out of energy, they can no longer transmit or receive messages. The 10 second simulation time provides ample duration for route discovery and formation.

\section{Simulation Setup of Group Mobility Models}

The group mobility simulation setup is similar to the random model setup with some additions. Here, we use our blockage models, group mobility and group connectivity patterns. We place and move the blockages in the field using a randomly generated pattern. We simulate them with a high loss factor (i.e., $L$ is very small). We choose these blockage settings to represent slow moving blockages, such as inclement weather or enemy jamming devices moving through a battlefield. Table II lists these additional parameters.

In the next two subsections, we describe the format of the graphs that we use to present our results in the following three subsections. Those results are based on the simulation parameters we have described above.

\section{Throughput Graph Description}

The throughput graphs in Figures 4, 6 and 8 contrast the performance of the modified MAC with power control to the unmodified MAC with fixed power transmissions. Each point depicts the percentage :

$$
100 * \frac{\text { TotalThroughput }(\text { Power Control } M A C)}{\text { TotalThroughput }(\text { FixedPower } M A C)}
$$

\footnotetext{
${ }^{3}$ We choose the seeds themselves arbitrarily. We produce twenty five sets of results for every simulation setup. The number of results is also arbitrary
}

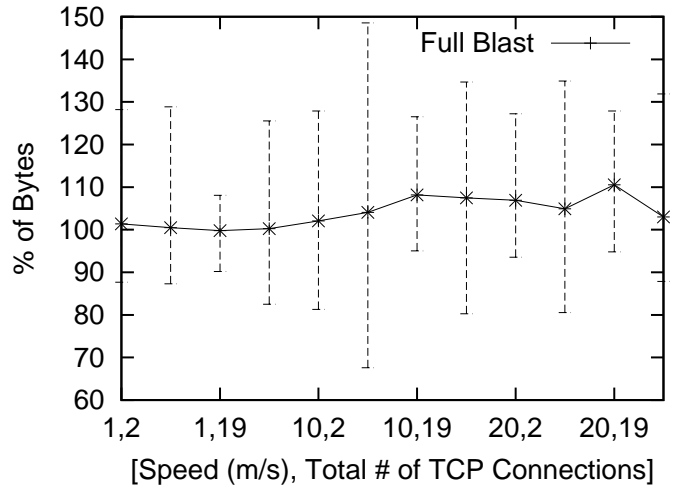

Fig. 4. Throughput Comparison Between The Two MACs (Random)

We define throughput as the total number of TCP session data bytes successfully transferred during the 10 seconds of simulation time for each run. Data bytes that are re-transmitted due to a loss in the data packets or acknowledgement messages or due to other reasons are accounted for (i.e., duplicate data bytes are not counted).

As described earlier, we conducted many simulations for each point using different random number generator seeds. Each point in the graphs shows the average value, and the vertical error bars show the maximum and minimum values. We vary both the speed $(1 \mathrm{~m} / \mathrm{s}, 10 \mathrm{~m} / \mathrm{s}$ and $20 \mathrm{~m} / \mathrm{s})$ and the total number of TCP connections instantiated along the horizontal axis. There is no particular scale across the horizontal axes - the goal of the variation along them is to show the robustness of power control across various situations.

\section{E. Energy per Byte Graph Description}

The "energy per byte comparison" graphs in Figures 5, 6 and 9 are similar to the throughput graphs except that each point in the graphs depicts the following percentage:

$$
\begin{aligned}
100 \quad & * \frac{\text { TotalEConsumed }}{\text { TotalThroughput }}(\text { Power ControlMAC) } \\
& * \frac{\text { TotalThroughput }}{\text { TotalEConsumed }}(\text { FixedPower } M A C)
\end{aligned}
$$

NS (version 2.1b6) only accounts for the energy consumed in receiving a message and the energy consumed in transmitting a message. Thus, the value of energy consumed represents the energy spent only in the simulated radio transceiver. This value assumes that the transceiver does not consume any energy when idle. Each node has an energy budget and when it exhausts this energy budget, it can no longer receive or transmit messages.

\section{F. Simulation Results with Random Mobility Models}

Figure 4 shows the improvement in total throughput of the power control MAC versus the unmodified MAC in simulations with random placement, mobility and traffic models. A value above $100 \%$ indicates that the power control MAC modifications achieved a higher overall throughput. 


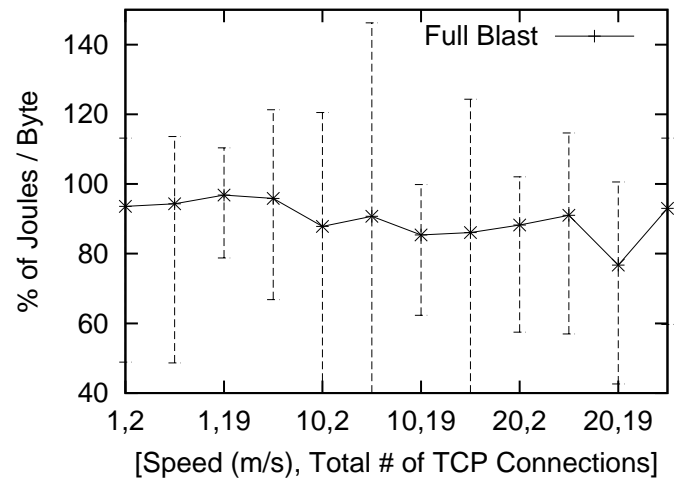

Fig. 5. Energy per Byte Comparison Between The Two MACs (Random)

Figure 5 shows the improvement in the energy consumed per transmitted byte. A value below $100 \%$ indicates that the power control MAC modifications consumed less energy overall.

The graphs show that the power control loop MAC achieves modest improvements in throughput and energy consumption. The variance is high, as indicated by the error bars. In simulations where communicating nodes happen to be at the maximum communication range distance between each other, the power control loop does not help. In fact, it consumes more energy and reduces throughput due to the extra control overhead transmitted in the message headers. However, in simulations where the communicating nodes happen to be close to each other, the power control loop successfully reduces the transmit power levels and reduces the total energy consumed. This can be due to both a reduction in the transmission energy consumed and due to a reduction in overall interference from other nodes. On average, it consumes about $10 \%$ less energy and improves the overall throughput of the system by about 5\% compared to the unmodified MAC. However, with our group mobility, traffic and blockage models, the power control MAC achieves significant improvements on throughput and energy consumption.

\section{G. Simulation Results with Group Mobility Models}

The simulations of group mobility and traffic patterns with blockage modeling produce more dramatic results. Figure 6 shows that the power control MAC offers roughly $15 \%$ higher throughput than the fixed power MAC. Also, the minimum error bars are mostly above the $95 \%$ line and the maximum error bars are much higher in these simulation results.

Similarly, Figure 7 shows that the overall energy consumption of the power control MAC is about $10-20 \%$ lower than the fixed power MAC. Again, the high error bars are much lower in this graph than in the random simulation graph and the low error bars are much lower. These results vary significantly from the simulations with random mobility models. We reiterate that it is important to test new algorithms in ad-hoc networking in conjunction with models of realistic deployment scenarios to assess the real impact of these algorithms.

As described in Section II, the power control loop initially starts off at the highest power setting when initiating communication with a node. During the course of subsequent communications, it ratchets the power setting down to a level just above

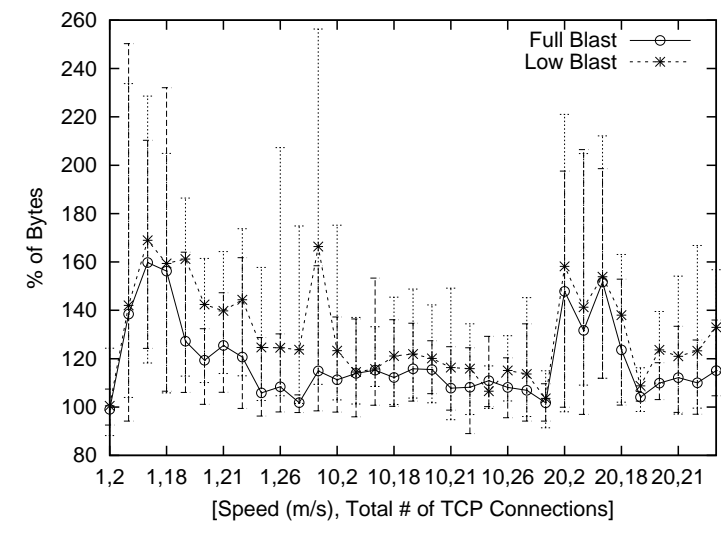

Fig. 6. Throughput Comparison Between The Two MACs

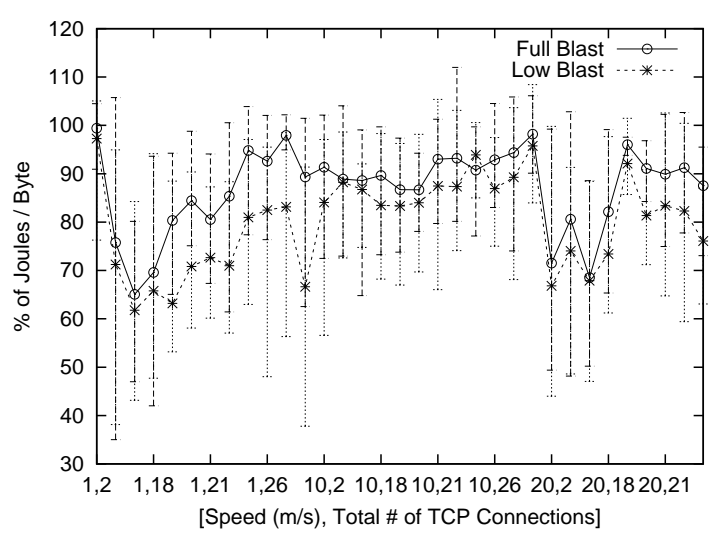

Fig. 7. Energy per Byte Comparison Between The Two MACs

which packet loss occurs (this shall be referred to as "full blast"). An alternative mechanism initially starts off at the lowest power setting and then ratchets up to the power level at which packets are accepted (this shall be referred to as "low blast"). The low blast mechanism has the advantage when nodes are close to each other since it avoids a "shouting match" problem. As expected, the above graphs show that the low blast algorithm has a lower energy consumption pattern and offers slightly higher overall throughput. However, it experiences an initial delay in communication between distant nodes when they ratchet up their transmission power levels until communication can be established.

Figures 8 and 9 are similar to the previous two graphs except that the horizontal axes are different. All the points on the graphs relate to a maximum node speed of $10 \mathrm{~m} / \mathrm{s}$ and a connection count of 28 TCP sessions. These graphs attempt to show how the benefits of the power control MAC change at various node densities; we vary the density of nodes by varying the simulated field area.

As we previously hinted, the main benefits of the power control loop result from two factors. Firstly, there is the reduction in energy consumed when transmitting at a lower power level, offset by the extra energy consumed in transmitting the power control information bits in the MAC message headers. This reduction in energy consumed means that there is more energy left for transmitting more bytes, thus also increasing the throughput of the system. Secondly, by reducing the power level of transmitted signals, the power control loop reduces the average interference. This helps to improve throughput because more data 


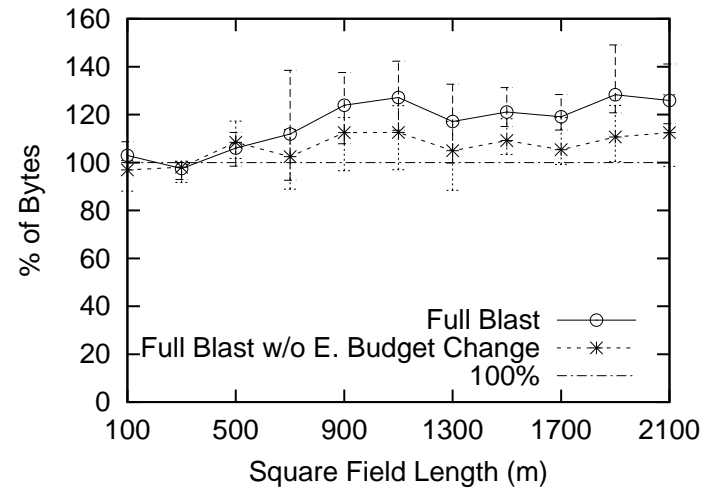

Fig. 8. Throughput Comparison Between The Two MACs (Density)

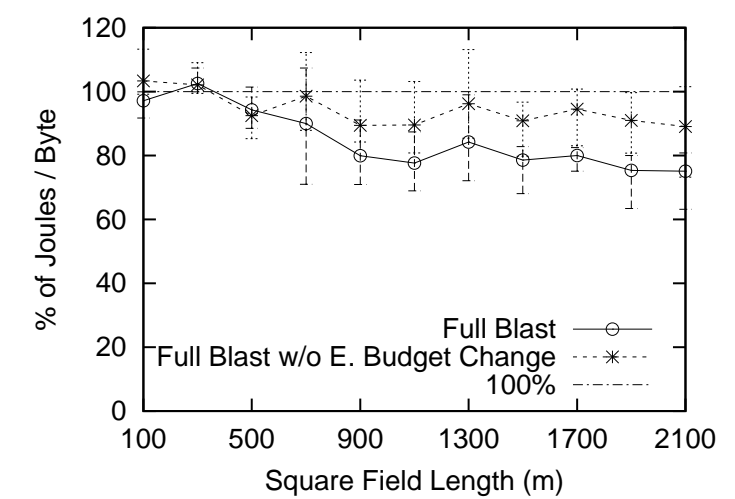

Fig. 9. Energy per Byte Comparison Between The Two MACs (Density)

can be in flight at the same point in time. This also translates into a reduction in energy consumed because there will be fewer transmissions that result in collisions / corruptions requiring retransmissions.

One way to assess the relative benefits of these two factors is to remove the first from the simulations. By not altering the energy consumed when transmitting packets (i.e., transmitting any packet costs the same energy as in the unmodified MAC), we can study the benefit obtained only from a reduction in interference. The "Full Blast w/o Energy Budget Change" lines in Figures 8 and 9 reflect the comparison between the power control MAC and the unmodified MAC when the transmit energy budget is fixed. Up to a square field length of $700 \mathrm{~m}$, the two lines are close. This means that in small areas, most of the benefits obtained by the power control are due to a reduction in interference. As the field size increases, the "Full Blast" line rises much higher then the other. For larger field sizes, the benefits are mostly due to a reduction in the transmit energy costs.

In this section, we have shown that the use of simulation models based on real usage applications rather than random models are beneficial. Without them, the true benefits or pitfalls of new algorithms or optimizations may not be realized. We have quantified the advantages of using power control loops for ad-hoc wireless networks. We have also discussed the effects that bring about these improvements in throughput and energy consumption.

\section{CONCLUSions}

Ad-hoc wireless networks will be employed in situations where the communicating nodes will not have access to wired power sources such as an electricity grid. These nodes vary in size, but many will be small units and thus will have very limited energy cells and energy scavenging abilities. A significant portion of a node's energy budget will be spent in communication. Thus it is important to explore new algorithms that minimize the energy cost of communication. We have proposed a methodology for achieving low power consumption in ad hoc networks. Power control at the MAC layer selects the minimum amount of transmit energy needed to exchange messages between any pair of neighboring nodes. We have described our distributed power control algorithm in detail and evaluated it by means of extensive and realistic simulation models. These realistic simulation models incorporate group mobility patterns, group traffic patterns and blockage models. We have shown that our power control loop improves energy consumption and throughput by $10-20 \%$ and $15 \%$, respectively.

\section{REFERENCES}

[1] JTRS, "Joint tactical radio system," http://www.jtrs.sarda.army.mil/.

[2] Tero Ojanperä and Ramjee Prasad, Eds., Wideband CDMA for Third Generation Mobile Communications, Universal Personal Communications. Artech House Publishers, 1998.

[3] William C.Y. Lee, Mobile Cellular Telecommunications Systems, McGrawHill, 1989.

[4] Lucent, "Wavelan specifications," http://www.wavelan.com.

[5] IEEE, "Medium access control (mac) and physical (phy) specifications," IEEE P802.11/D10, Jan 1999.

[6] B. Narendran, J. Sienicki, S. Yajnik, and P. Agrawal, "Evaluation of an adaptive power and error control algorithm for wireless systems," IEEE ICC, vol. 1, pp. 349-355, June 1997.

[7] T.J. Kwon and M. Gerla, "Clustering with power control," IEEE MILCOM, vol. 2, pp. 1424-1428, Nov 1999.

[8] R. Ramanathan and R. Rosales-Hain, "Topology control of multihop wireless networks using transmit power adjustment," IEEE INFOCOM, vol. 2, pp. 404-413, March 2000.

[9] J. Broch, D.A. Maltz, D.B. Johnson, Y.C. Hu, and J. Jetcheva, "A performance comparison of multi-hop wireless ad hoc network routing protocols," MOBICOM, October 1998.

[10] V. Bharghavan, A. Demers, S. Shenker, and L. Zhang, "Macaw: A media access protocol for wireless lans," SIGCOMM, September 1994.

[11] David J. Goodman, Wireless Personal Communications Systems, Wireless Communications Series. Addison Wesley, 1997.

[12] C. Graff, M. Bereschinsky, M. Patel, and L. F. Chang, "Application of mobile ip to tactical mobile internetworking," IEEE MILCOM, vol. 1, pp. 409-414, Oct 1998.

[13] J. A. Stine and G. Veciana, "Tactical communications using the ieee 802.11 mac protocol," IEEE MILCOM, vol. 1, pp. 575-582, Oct 1998.

[14] R. Antkiewicz, A. Manikowski, A. Najgebauer, and T. Nowicki, "A computer simulator of a tactical communication system," IEEE MILCOM, vol. 1, pp. 893-897, Oct 1998.

[15] P. Johansson, T. Larsson, N. Hedman, B. Mielczarek, and M. Degermark, "Scenario-based performance analysis of routing protocols for mobile adhoc networks," MOBICOM, pp. 195-206, 1999.

[16] NS-2, "Network simulator," http://www-mash.cs.berkeley.edu/ns/, 2.1b6.

[17] J. Broch, D.B. Johnsson, and D.A. Maltz, "The dynamic source routing protocol for mobile ad hoc networks," Internet Draft, pp. draft-ietf-manetdsr-00.txt, March 1998. 